Revue d'histoire de l'Amérique française

6. REVUE D.HISTOIRE DE L'AMÉRIQUE FRANÇAISE

\title{
Deux lettres de Louis-Joseph Papineau à J.-J. Girouard
}

Volume 15, numéro 2, septembre 1961

URI : https://id.erudit.org/iderudit/302116ar

DOI : https://doi.org/10.7202/302116ar

Aller au sommaire du numéro

Éditeur(s)

Institut d'histoire de l'Amérique française

ISSN

0035-2357 (imprimé)

1492-1383 (numérique)

Découvrir la revue

Citer ce document

(1961). Deux lettres de Louis-Joseph Papineau à J.-J. Girouard. Revue d'histoire de l'Amérique française, 15(2), 283-288. https://doi.org/10.7202/302116ar d'utilisation que vous pouvez consulter en ligne.

https://apropos.erudit.org/fr/usagers/politique-dutilisation/ 


\section{DOCUMENTS INÉDITS}

Copie de deux lettres de Louis-Joseph Papineau à M. J.-J. Girouard, don fait à l'Institut par Mme Albert Décarie, née Jeanne Girouard. Nous les publions en respectant scrupuleusement l'orthographe, même les ratures. Il se peut que certaines parties de ces lettres, surtout de la première, aient déjà été publiées. Ces extraits n'ont pu être tirés que d'une copie, puisque nous possédons l'original.

$$
\text { N.D.L.D. }
$$

\section{Mon Chèr Monsieur Girouard}

De Monté-Bello, 30 Mai 1855

Je commence par vous souhaiter \& à Madame, à votre famille $\&$ aux amis du voisinage, santé \& contentement; à vous prier d'excuser le délais écoulé depuis la réception de votre lettre du dix huit présent mois; et à vous expliquer les causes de ce délais. J'attendais de jour en jour, Amédée de Montréal, et lui attendait de jour en jour que notre chemin de fer que nous désirons si fort, non le vôtre, que vous désirez si peu, recommençat à rouler comme l'automne dernier, de Carillon à Grenville. Les Directeurs à Montréal espéraient en venir à un accomodement avec les propriétaires limitrophes. Ils n'y ont pas reussi : et Amédée pour éviter la fatigue du longsault, plus grande que jamais par la désorganisation du roulage sur la voie publique dintes les propriétaires cnt⿳亠丷厂犬 ayant vendu (stages) voitures et chevaux, il a pris enfin la résolution de venir par chemin de fer par voie de Rouse's Point, Ogdensburg et Byetown, et de là ici par le bateau à Vapeur: 150 lieues, en place des Vingt cinq de la route directe Et il la trouve tellement préférable qu'après m'avoir donné quatre jours ici, il est reparti par la même voie avec ses sœurs pour Montréal. Si nous avions notre chemin de fer, nous serions en deux heures à St Benoit et nous voisinerions; nous serions en trois heurres à Montreal et ne resterions pas dans notre impénétrable solitude, où nous nous rouillons et devenons à vue d'œil de parfaits Algonquins. Il y a toute apparence que nous serons devenus tels irrémédiablement, avant que les canons Russes soient tous pris \& fondus en rails, ce quil nous faudra attendre sans doute, avant que toute entreprise de chemin de fer qui n'aura pas à sa disposition le pillage de la Province, comme le grand tronc, puisse se continuer. Vous voyez donc qu'à part ma loyauté toujours la même pour la 
Mère Patrie, je suis interressé à la défaite des Russes. J'attendais donc Amédée pour savoir ce qui avait été fait du monument que vous aviez fait déposer sur les restes de mon chèr Père, lorsqu'il les avait dernièrement fait relever. Mon intention avait été de me trouver à Montréal à cette occasion, mais les médecins consultés sur les précautions à prendre, conseillèrent de le faire de suite avant que les chaleurs ne rendissent cette opération beaucoup plus difficile, si comme il y avait lieu de le craindre du laps de tems écoulé depuis 1841, la corruption du corps et des cercueils, avait été très grande. Sur cet avis Amédée obtint de suite du Juge et de Monseigneur le Coadjuteur de Montréal, l'autorisation de faire faire cette exhumation \& de la bonté de Monsieur le Supérieur, la permission de déposer le Corps renfermé avec ses premiers cercueils dans un troisième en Zinc Soudé, pour qu'il n'enèmanât point de mauvaise odeur, dans les voutes de l'église paroissiale, jusqu'à ce qu'il puisse être apportée ici. Si le chemin de fer avait glissé sur ses rails, il aurait pû être apporté ici par les bateaux à vapeurs et les locomotives. Il a fallu renoncer à l'idée de l'apporter par les secousses du roulage. Alors Amédée a projetté l'apporter par un des petits bateaux à vapeurs de touage, mais leurs propriétaires lui ont dit que les travaux du Grand tronc avaient excessivement gâté la navigation du St Laurent à $\mathrm{S}^{\text {te }} \mathrm{Anne}$; que deux de ces bateaux y avaient échoué et n'avaient été dégagés qu'après de longs délais et que deux de leurs barges y avaient été brisées; qu'en conséquence ils lui conseillaient d'attendre aux eaux moyennes, où ces dangers cesseront, pour venir avec ces restes vénérés, ce qu'il fera sous un mois à peu près.

Lorsque j'eus l'extrême douleur de perdre ici mon chèr Gustave, expiré avec la plus entière resignation à l'ordre de la Providence, à l'âge de vingt et un ans, dans les bras de ses inconsolables parents, Amédée accouru pour partager nos souffrances et assister aux funérailles de ce chèr enfant, me proposa de bâtir une chapelle non loin de ma demeure \& qui dût servir de tombeau de famille. Oui lui dis-je je dois le faire. Je prends racine ici. Je ne m'eloignerai probablement pas du lieu où repose celui que j'aurais dû devancer. J'y réunirai le corps de mon Père et bientot après mes restes seront déposés auprès. Mon chèr Père a vaincu tant d'obstacles, surmonté tant de fatigues, fait tant de dépenses pour commencer l'établissement de cette Seigneurie, qu'il est bien, que nous y reposions les uns après les autres, aux pieds d'un homme qui a tant fait pour son pays et pour sa famille. Que son souvenir nous soit une leçon qui nous profite, et aux enfans de nos enfans. J'ai donc durant les deux été derniers travaillé à cette chapelle qui n'est pas 
considérable, mais tant d'autres constructions, plantations \& défrichemens se suivent en même tems, qu'avec des ressources moyennes, l'on ne peut aller qu'un peu plus lentement que l'on ne voudrait. Elle est presque terminée; d'un stile gothique très uni, un peu agreste; tel qu'il me parait bien convenir au local et à la destination. Nous ne pouvons arriver à la maison ni en sortir sans passer tout près de notre dernière \& permanente demeure, située dans un joli boccage qui s'embellira avec la croissance de sa jeune forêt et les plantations additionnelles projettées; à trois arpens de la résidence transitoire et passagère, à cinq de l'église paroissiale dont vous connaissez la situation. L'hiver dernier j'ai eu la douleur d'y ouvrir et fermer la première tombe, pour le premier de mes petits fils, bel enfant d'un an emporté par sa première maladie. Le pauvre Père l'a apporté en fevrier dernier pour le déposer dans la Chapelle dont le premier il avait formé le projet et tracé le plan presque constemment suivi. La seconde s'est ouverte \& fermée sur le corps de mon Chèr Gustave ces jours derniers pendant que Amédée était avec moi. La troisième est construite pour recevoir bientôt les restes de mon chèr Père. Mais elle est béante, \& j'y puis tomber avant qu'ils arrivent. Que la volonté de Dieu soit faite en tout \& partout. Je puis laisser la vie sans regrets. J'y ai éprouvé trop de pertes désolantes pour y tenir fortement. Les avertissemens se multiplient trop rapides, pour que je puisse être surpris. De 22 écoliers entrés en Rhétorique en 1802 je suis le seul survivant. Mon ami $\mathrm{M}^{\mathrm{r}}$ Antoine Parant ci devant supérieur du Séminaire de Québec, était le seul de cette classe sur le bras duquel je pusse m'appuyer. Il n'avait qu'un an plus que moi. Mon ami \& parent de cœur et d'enfance, avec qui j'ai été élevé comme frère, Louis Viger n'ayant aussi qu'un an plus que moi, tombe et je l'apprends pendant que je vous écris. Je dois me hâter de faire le peu de bien que je pourrai, puis aller rejoindre tant et tant de personnes que j’ai tendrement chéries.

Si Dieu me prête vie jusque là, j'assisterai à la Bénédiction de cette Chapelle par mon Evêque Diocésain; dans les premiers jours d'Août. Je l'ai prié de venir faire cette cérémonie et d'en fixer le tems, il m'a donné par sa reponse reçue tout récemment, Ceux des dix premiers jours d'Août qui me conviendraient le mieux, et je lui écris aujourd'hui même pour le prier de déterminer plus particulièrement celui qui lui conviendra, pour que je puisse l'indiquer à ceux des parents et amis qui y viendront. Vous assistiez le $137^{\text {bre }} 1821$, avec ces bons Amis que nous n'avons cessé de regretter, Messieurs Felix \& Dumouchel à la Bénédiction de la première église paroissiale. Nous sommes Bien peu de survivans de cette première réunion. Vous assisterez je vous en 
prie avec Madame votre épouse, $\mathrm{M}^{\mathrm{r}}$ \& Madame Dumouchelle $\mathrm{M}^{\mathrm{r}}$ \& Madame LeMaire à notre seconde bénédiction. Nous étions alors tous jeunes, peu éprouvés, peu attristés, pleins d'entrain, d'avenir, de projets; que de mécomptes. Néanmoins c'est la condition que l'ordre de la providence a établi dans tous les tems pour tous les hommes. Soyons soumis. Au plaisir de nous voir réunis de nouveau, se mêleront des souvenirs \& des regrets pour tous ceux que nous avons perdus; mais avec les regrets se mêle le plaisir de parler de leurs mérites, de leur amabilité, de leur affection pour nous. Nous rajeunissons un peu en parlant du bon vieux tems, des bons vieux amis. J'attendais la fin de ma correspondance avec Monseigneur de Bye-town pour vous faire cette prière, que je vous fais aujourd'hui et que je n'ai encore adressée à personne de la famille. $\mathrm{M}^{\mathrm{e}}$ Dessaulles et mes frères \& les frères \& soeurs de mon épouse \& des neveux et des nièces, et ma bonne belle soeur Angelle, sont ceux que j'espère réunir pour cette occasion avec les amis de $S^{t}$ Benoit. D'ici là le jour précis sera déterminé et je vous en préviendrai. Je savais que je devais à votre sincère amitié pour mon Père \& pour toute sa famille le monument si judicieusement choisi d'un cube, symbole de la solidité de son caractère, de ses principes, de sa persévérance à suivre les études \& les entreprises utiles qu'il a successivement commencées \& continuées tant qu'il a respiré. Je devais vous demander que la Pierre tumulaire suivit les restes chèrs $\&$ vénérés auxquels vous l'aviez consacrée. Vous me prévenez, parce que le moment de la dédicace de la Chapelle ou ils seront portés et du service funèbre pour les âmes des parents décédés et en particulier de ceux qui y sont déposés, qui y sera fait, n'était pas fixé. Votre partiale amitié a étendu trop loin les justes éloges que vous donnez à la Mémoire, aux vertus publiques \& privées qu'il mon Père eut aux services qu'il a rendus à la nationalité, à la patrie, à tant et tant de ses concitoyens, en les étendant à la famille, qui a marché sur ses traces il est vrai, mais d'un pas moins sur et moins heureux que lui. Il paraitrait donc un peu outré que dans une chapelle de famille \& privée quelqu'un de ses descendans eut écrit, Laboribus ac Sobole clarior. Mais les A.P.P. est un premier et suffisant correctif. Ensuite le Sobole ne s'applique pas à sa seule postérité, mais à sa postérité Morale, patriotique, éclairée, qui sont tous les disciples que son exemple, et ses leçons ont fait naitre à la vie politique, à la défense énergique des droits de son pays, tous ceux qui sont tombés, tous ceux qui ont souffert pour cette sainte cause dont il a été le doyen; ceux qui font autant d'honneur au Canada \& au Notariat comme $\mathbf{M}^{\mathrm{r}}$ Girouard, et 
autres Notaires en grand nombre depuis ce bon $\mathrm{M}^{\mathrm{r}}$ Gagnier le plus ancien de ses clercs, jusqu'à J.B. Trudeau \& André Papineau les derniers qu'il a formés, tous ceux que ses encouragemens \& ses conseils ont aidé à se distinguer à leur tour, sont inclus dans l'expression. Si j'avais été présent c'eut été à moi à remplir le pieux devoir, que je n'aurais pu remplir aussi parfaitement bien, comme vous l'avez fait, sans vos conseils, mais ils ne m'auraient pas fait défaut. J'accepte donc avec reconnaissance l'Offre que vous me faites et que j'étais à la veille de vous demander, que le monument fut inséparable du corps sur lequel il a été posé. Il est demeuré au Cimetierre sur la fosse vide du corps de mon Père mais dans laquelle sont les restes reduits en poudre de cinq enfans morts en bas âges. Voila la vie de l'homme, ses amertumes, ses inscrutables mistères, plus de la moitié de ce qui nait d'hommes, dévorés par la mort, avant qu'ils soient parvenus à cinq ans ! O Abimes!

Faire du bien \& beaucoup et durable comme vous avez su le faire, Mon cher $\mathrm{M}^{\mathrm{r}}$ Girouard par la noble fondation que vous venez de terminer, voilà un monument qui consacre à toujour votre mémoire et y attache les prières et les bénédictions de tous ceux qui y seront instruits et soulagés. Adieu.

Votre ami bien sincère et reconnaissant.

LJPapineau

Mon chèr Monsieur Girouard

Monté Bello 27 juillet 1855

Depuis que je vous ai écrit pour vous dire que mon épouse ma famille et moi désirions fort que vous nous fissiez l'amitié de nous venir voir avec nos autres amis de $\mathrm{S}^{\mathrm{t}}$ Benoit, Monseigneur de Byetoym a choisi le premier mardi d'août prochain pour le jour où il bénira notre petite chapelle funeraire et de famille. Il y a trente trois ans que dans cet établissement alors à son berceau, vous êtes venu avec tant de bons amis, dont si peu survivent, assister à la bénédiction de l'église paroissiale. Depuis lors la même cérémonie s'est répétée pour deux autres églises paroissiales dans la ci-devant Seigneurie de la Petite Nation. Aucune réunion ne peut se répéter pour nous, à nos âges, comme celles qui avaient lieu alors, quand des natures aussi heureuses que celles de mon Père de Messieurs Felix \& Dumouchel donnaient l'ordre, le signal \& l'exemple d'être gais. Depuis lors ils nous ont laissés \& la gaité a été ensevelie avec eux. Les années se sont accumulées \& bien plus encor les Deuils \& les chagrins. Néanmoins quand de Anciens amis comme vous \& moi se revoient, ce sont des jours de rajeunissement, puisque nous avons peu à 
dire des évènemens contemporains \& beaucoup à dire des évènemens passés; nous nous fesons illusion pendant quelques instants en parlant du bon vieux tems, ou nous éprouvions bien des contrariétés tout comme depuis, mais où pour chaque contretems qui nous chagrinait, mille espérances nous déridaient. Venez voir vos ainés \& les consoler, Madame Dessaulles, Madame Benjamin Papineau, Mon épouse et moi, ce sera oeuvre de charité $\&$ d'amitié dont tous, nous serons bien reconnaissans. Si vous ne veniez qu'avec des hommes, les voitures publiques suffiraient. Mais si vous ajoutez au plaisir que nous donnera votre bonne compagnie, celui que nous donnerait votre bonne compagne, si elle veut bien nous faire cette amitié, où quelqu'autre Dame, la voiture publique est bien dure \& encombrée, les chevaux bien rosses, les chemins du long sault bien pierreux, et ce trajet bien fatigant. Au lieu que si vos bonnes voitures vous amenaient avec $\mathrm{M}^{\mathrm{rs}} \& \mathrm{D}^{\mathrm{es}}$ Dumouchel \& Lemaire, de manière à arriver à Grenville à Deux heures, vous seriez ici à quatre sans avoir éprouvé d'incommodité. Si vous avez l'obligeance de me prevenir du jour de votre arrivée, j'irai avec mes voitures vous prendre au débarquement chez Major. Le Mercredi nous aurons dans la chapelle bénie de la veille, un service à la mémoire des Chèrs Parents décédés.

Dans l'espérance de vous voir alors je vous prie de vous préparer à me répondre sur l'affaire suivante dont je vais vous entretenir. Vous avez me dit-on fait l'inventaire de ce bon brave héroïque Docteur Chénier. Sa Mère était son héritière. Elle a légué tout ce qu'elle avait à sa Belle fille femme du Capitaine Chénier de Longueuil. Celle-ci depuis son veuvage s'est faite religieuse à Longueuil; mais avant de faire ses voeux, elle a fait donation de Vingt-cinq Louis à elle dûe en vertu du legs de sa Belle Mère par la Succession du Docteur Chénier, à ses deux belles sœurs qui demeurent ici, l'une épouse de $\mathbf{M}^{\mathrm{r}}$ Louis Thibeaudeau cultivateur peu à son aise, et l'autre épouse du Père Lalonde qui sont dans une grande pauvreté. Cette somme toute modique qu'elle est, est importante pour ces deux personnes tout à fait recommendables. Je vous demanderai donc quand j'aurai le plaisir de vous voir, si elles ont quelques chances de la recouvrer \& à qui elles devront s'adresser pour l'obtenir.

Adieu mon cher Monsieur Girouard; souhaitant que la présente vous trouve avec Madame votre épouse et votre famille ainsi que Messieurs Dumouchelle \& Lemaire, leurs Dames \& leurs familles en santé \& contentement, je vous dis à tous au revoir. 